\title{
Die Lage der Niere beim menschlichen Embryo und die der Hufeisenniere.
}

\author{
Von
}

Prof. Dr. M. Zondek.

Die Lagebeziehung der Nierenanlagen zur Wirbelsäule und der Bifurkation der Aorta in die Arteriae iliacae hat $\mathrm{Ha} \mathrm{c} \mathrm{h}^{1}$ ), an menschlichen Embryonen bis ungefähr zur 20. Woche eingehend untersucht. Am Embryo von $7-8 \mathrm{~mm}$ sah er den Ureter sich aus. der lateralen Seite des Urnierengangs abzweigen. Der Ureter wendet sich dorsalwärts, bis er die Arteria umblicalis trifft. Hier teilt er sich in je einen oberhalb und unterhalb der Arterie verlaufenden, kurzen, starken Ast. „Die Arteriae iliacae ziehen unter rechtem Winkel von der Aorta ab, die sich in die Arteria caudalis fortsetzt. Die beiden Nierenanlagen liegen hier also ganz dicht aneinander, jede an ihrer Seite der Mittellinie, von den Arterien umschlossen."

Bei einem Embryo von ca. $2 \mathrm{~cm}$ Länge ist die Nierenanlage schon zum größten Teil kraniell von der Arteria iliaca gelegen. Ferner erscheint mir besonders bemerkenswert, daß ,,der kranielle Teil stark in dorsaler Richtung und lateral gezogen ist, während' die kaudalen Pole näher aneinander mehr ventral liegen“.

An Embryonen bis zur 20. Woche verbleibt der untere Nierenpol in der Nähe des oberen Randes des vierten Lendenwirbels, während der obere Nierenpol schließlich die elfte Rippe erreicht hat. Der obere Pol hat also jetzt bereits die Lage wie beim Erwachsenen, während der untere Pol verhältnismäßig weit kaudal zurückgeblieben ist.

1) E. Hauch, Ueber die Anatomie und Entwickelung der Nieren. Anat. Hefte. Beiträge und Referate zur Anatomie und Entwicklungsgesch. Herausg. von Fr. Merkel und R. Bonnet. Bd. XXII. Heft 69. 1903. 
Beachtenswert erscheint mir auch die Lagebeziehung des unteren Pols der Niere zur Hölue der Bifurkation der Aorta. Sie liegen beide zuı ̈̈cns! in gleicher Höhe. Die Bifurkationsstelle der Aorta behält diese Lage ungefähr auch bei der weiteren Entwicklung bei, wälırend der untere Pol jeder Niere höher zu liegen kommt.

Gleichzeitig mit der Verschiebung der Niere nach oben erfolgt eine Drehung der Niere um ihre Längsachse. Bei einem Embryo von 7-8 $\mathrm{mm}$ mündet der Ureter in die vordere Wand der Nierenanlage. Bei der weiteren Entwicklung dreht sich die Niere um ihre Längsachse nach innen. Bci einem 6 Wochen alten Embryo nimmt die Niere eine frontale Stellung ein, und der Sinus der Niere ist nach der Mittellinie hin gerichtet. Später erfolgt wieder die Drehung nach auBen, und die Niere gewinnt scliließlich die endgültige Stellung, die etwa in der Mitte zwischen frontaler und sagittaler Stellung liegt.

Die physiologischen Verschiebungen der Nieren können durch irgend eine Störung gehemmt werden. Es entsteht die kongenitale Heterotopie des Organs. Die Niere bleibt dann an einer abnorm tiefen Stelle zurück. Ihre frontale Achse liegt in einer zur Frontalachse des Körpers abnormen Stellung. Unter Hinweis auf meine früheren Ausführungen ') sei hier kurz nur folgendes bemerkt: Die Heterotopie kann auf einer oder auf beiden Seiten vorhanden sein. Bei Heterotopie einer Niere ist gewöhnlich die andere an normaler Stelle gelegen und normal, gelegentlich aber hypoplastisch oder überhaupt nicht vorhanden. Ferner können beide Nieren kongenital heterotop sein. Hierbei müssen wir zwei verschiedene Arten unterscheiden: Beide heterotopen Nieren liegen selbständig auf jeder Seite. Das kommt sehr selten vor; S t r u b e hat aus der Literatur im ganzen nur 4 solche Fälle zusammengestellt. Oder die kongenital heterotopen Nieren sind miteinander verwachsen. Dabei ist wiederum zweierlei mögl ch: Die Nieren sir:d in ganzer Ausdehnung zur ,Kuchenniere“ verschmolzen. Solche Beobachtungen sind sehr selten. Oder die Nieren sind nur an ihren unteren Polen durch eine Gewehsbrücke miteinander verwachsen. Man nennt diese Anomalie Hufeisenniere, ren arcuatus. Die Hufeisenniere kommt verhältnismäßig häufig vor. Nach der Zusammenstellung von $\mathrm{C}$ h a $\mathrm{rlier}$ und $\mathrm{G} e \mathrm{r}$ ard kamen

1) Die Topographie der Niere und ihre Bedeutung für die Nierenchirtrgie. Verl. A. Hirschwald, Berlin, 1903, S. 80--95. 
bei einem Material von 68989 Fällen eine Hufeisenniere auf 1000 Sektionen, und nach G. Marzynskys Berechnung auf 29384 Sektionen 43 Hufeisennieren, also je eine auf 683 Sektionen. Die Hufeisenniere ist ferner infolge ihrer Nähe zur vorderen Bauchwand traumatischen Einwirkungen von außen sehr ausgesetzt. Dies trifft insbesondere auch für die Ureteren zu, die von der vorderen Wand der Nieren abziehen und über die die Schenkel verbindende Brücke gewissermaßen hinüberklettern. Die Hufeisenniere ist đaher verhältnismäßig oft erkrankt. Es ist also von großem praktischem Wert, ihre Form und Lage genau zu kennen, zumal da wir gelernt haben, in vivo einen mehr oder weniger großen Teil der Niere durch die Bauchdecken hindurch abzutasten oder im Röntgenbild darzustellen. Da die Hufeisenniere durch Verschmelzung der Nieren an ihrem unteren Pol im embryonalen Leben entsteht, werden wir die Anomalie besser verstehen, wenn wir ihre Form und Lage mit derjenigen der Niere in verschiedenen Stadien der embryonalen Entwicklung vergleichen. Die vorliegenden Untersuchungen wurden an 8 menschlichen Embryonen und ferner an 4 Hufeisennieren ausgeführt, die mir Herr Kollege $\mathrm{Max} \mathrm{Koch}$, Prosektor am Krankenhaus am Urban, freundlichst zur Verfügung gestellt hat.

Ich habe die Entfernung der Innenränder beider unteren und die beider oberen Pole voneinander gemessen, ferner an jeder einzelnen Niere, die Breite in der Nähe des Hilus, und die Lagebeziehung ihrer Längsachse zu derjenigen der Wirbelsäule bestimmt.

Im allgemeinen ist beim Erwachsenen die linke Niere etwas länger als die rechte. Bei den 8 Embryonen waren in 4 Fällen die linke, in den anderen 4 Fällen die rechte Niere die größere. In der Höhe des Hilus gemessen war in 5 Fällen die rechte, in 3 Fällen die linke Niere breiter. Bei den Embryonen von 11, $24,34 \frac{1}{2} \mathrm{~cm}$ Steiß-Scheitelbeinlänge lagen die beiden unteren Nierenpole etwas tiefer als die Bifurkationsstelle der Aorta; bei den Embryonen von 39 und $41 \mathrm{~cm}$ Steiß-Scheitelbeinlänge liegen beide untere Nierenpole oberhalb der Teilungsstelle der Aortâ; bei Embryonen von 15 und $28 \mathrm{~cm}$ der linke untere Pol in gleicher Höhe, der rechte etwas tiefer; beim Embryo von $321 / 2 \mathrm{~cm}$ der rechte in gleicher Höhe, der linke etwas höher. In 6 Fällen die linke, in 2 Fäilen die rechte aber etwas höher. 
Die Lage der Niere beim menschlichen Embryo usw.

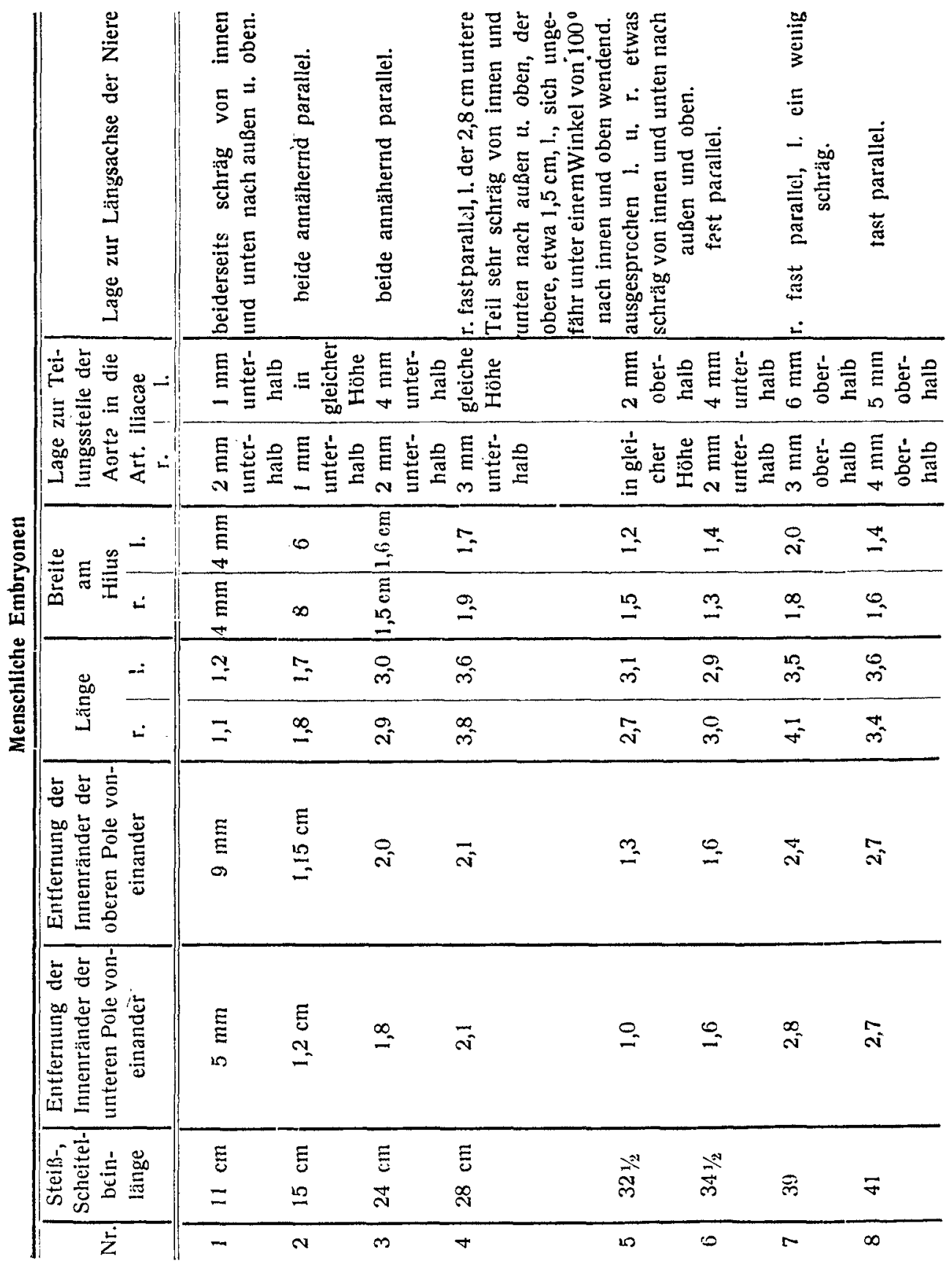




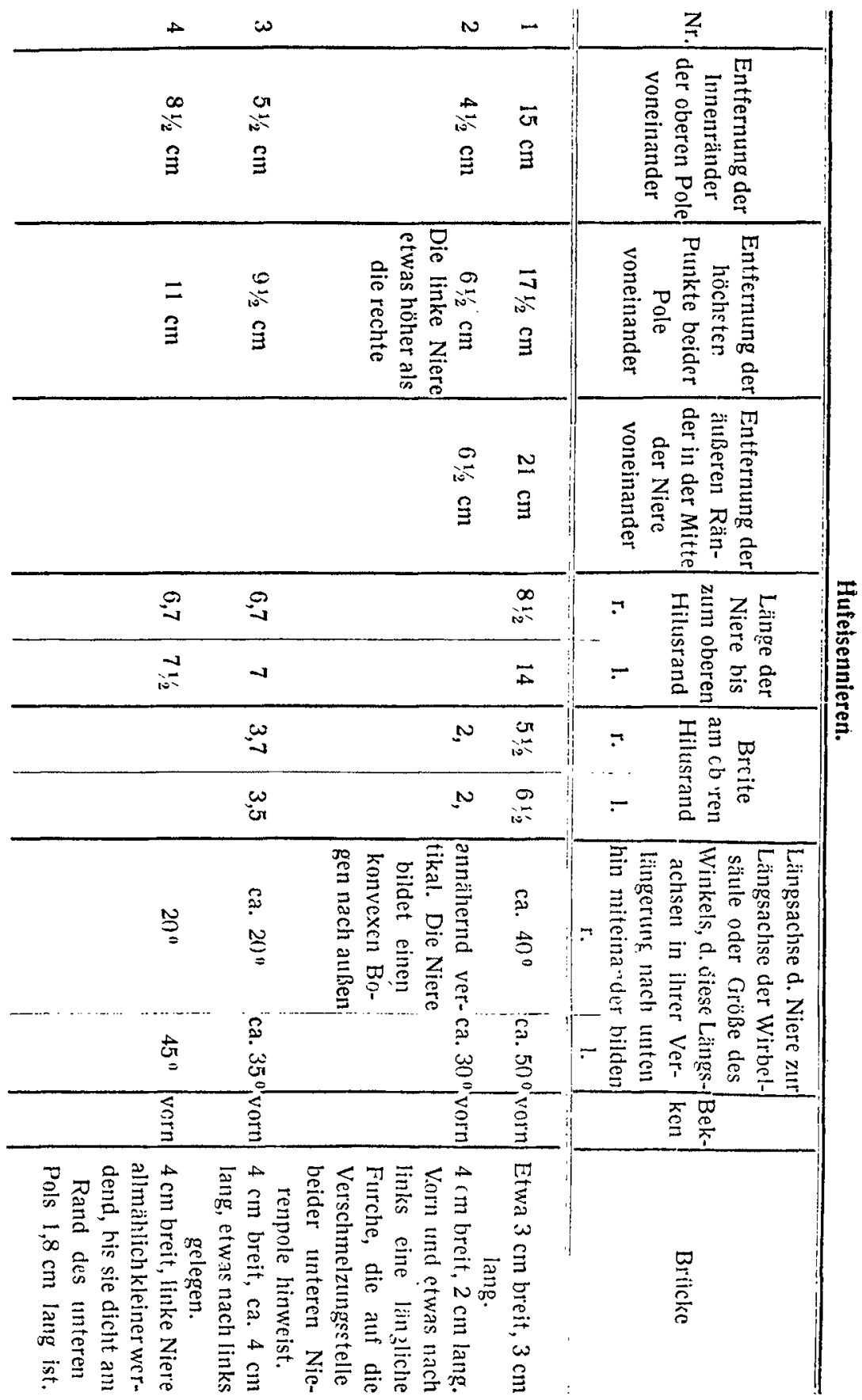


Bei den Embryonen ist am oberen Nierenpol ein medialwärts vorspringender Teil vorhanden, der von der Nebenniere überlagert ist. Ist auch dieser medialwärts gerichtete Teil am oberen Pol im Verhältnis zur übrigen Niere klein, so beeinflußt er doch đie Lage der Längsachse der Niere im Verhältnis zur Längsachse der Wirbelsäule. Berücksichtigt man ihn nicht, sondern betrachtet die Niere nur soweit, als sie nicht von der Nebenniere bedeckt ist, dann sind die Längsachsen fast immer so gerichtet, daß sie in ihrer Verlängerung nach unten einen spitzen Winkel bilden. Betrachtet man aber die Niere nach Abpräparierung der Nebenniere, dann war im wesentlichen nur im Stadium von 11 und $321 \% \mathrm{~cm}$ SteibScheitelbeinlänge die Längsachse der Niere nach innen und unten gerichtet, in den übrigen Fällen aber annähernd parallel der Längsachse der Wirbelsäule gelegen.

$\mathrm{H} \mathrm{auch}$ fand beim Embryo von $2 \mathrm{~cm}$ Länge ebenfalls die kaudalen Pole beider Nieren näher aneinander gelegen als die kraniellen Pole. Bei dem $7-8 \mathrm{~mm}$ langen Embryo lagen die Nierenanlagen dicht nebeneinander, und der Ureter drang'in die vordere Wand der Niere ein. Wir müssen also die Verschmelzung beider Nieren an ihren unteren Polen zur Hufeisenniere für ein selır frühes Stadium der embryonalen Entwicklung annehmen. An den 4 hier beschriebenen Hufeisennieren sind die Schenkel von innen und unten nach außen und oben gerichtet. Nur in Präparat 2 ist die Längsachse der rechten Niere parallel zur Längsachse der Wirbelsäule. Die Brücke zwischen den Schenkeln ist $3-4 \mathrm{~cm}$ breit. In allen Fällen liegen die Nierenbecken mehr vorn an den Organen, als normal.

Diese Feststellungen haben nicht nur ein theoretisches, sondern auch praktisches Interesse. In einem Fall konnte ich auf Grund der angefülhrten Merkmale vor der Operation durch Palpation und Röntgenbefund die Diagnose auf Hufeisenniere stellen; weitere klinische Symptome ergaben Vereiterung und Stein in linken Schenkel der Hufeisenniere. Der festgestellten Anomalie entsprach das operative Vorgehen, das von Erfolg war. 